\title{
Internal Picture of the Disease as a Success Factor of Postoperative Neurorehabilitation in Patients with Pituitary Adenoma
}

\author{
A.M. Domracheva, I. A. Safonova, A. O. Denisenko, D.I. Sozaeva, S.B. Berezhanskaya, A.A. Afonin, \\ I.V. Panova, S.Kh. Dombayan, T.A. Afonina \\ Federal State Budgetary Educational Institution of Higher Education "Rostov State Medical University" of the \\ Ministry of Health of the Russian Federation \\ Rostov-on-Don, Nakhichevan lane, 29, 344022 \\ Russia
}

Received: February 7, 2021. Revised: August 3, 2021. Accepted: August 30, 2021. Published: September 1, 2021.

\begin{abstract}
In order to study the perception of patients operated on for pituitary adenoma, their disease, the types of response to the disease, as well as the influence of the type of internal picture of the disease $((\mathrm{VKB})$ on the success of the treatment and rehabilitation process, 52 patients ( 24 men, 28 women) aged from 32 to 46 years old (mean age $-37,7 \pm 1,2$ years), operated on for pituitary adenoma Analysis of medical records, interviews, assessment of the patient's condition using a number of rating scales and psychodiagnostic techniques to determine the type of attitude to the disease revealed in patients a high level of nosogenic stress reactions and a significant predominance of a maladaptive attitude towards an intrapsychic disease $(92,3 \%)$. The presence of the dominant maladaptive type of VKB in patients significantly reduces the socio-psychological adaptation in the postoperative period. Under these conditions, in the process of forming a strategy of postoperative neurorehabilitation for the successful restoration of the psychosocial status of patients with pituitary adenomas, it is necessary to take into account the nature of maladaptive personality reactions.
\end{abstract}

Keywords - pituitary adenoma, internal picture of the disease, neurorehabilitation, neurooncology.

\section{INTRODUCTION}

$\mathrm{T}$ HE development and improvement of the system of complex neurorehabilitation procedures, taking into account the individual and personal characteristics of neurooncological patients, is one of the urgent tasks of modern neurology and clinical psychology.

Modern medicine is characterized by the introduction of ideas and methods of medical psychology into the clinic of various diseases, since when they occur, there is always, in the words of K.K. Platonov, "complex circular dependences of the mental and somatic in the type of a circle, and in some cases, a vicious circle", with the mutual transition of psychogenia to somatogeny and vice versa. Not only psychopharmacological, but also psychotherapeutic influences on his psyche can help the patient to get out of such a vicious circle "with the aim of eliminating painful symptoms, changing the attitude to his disease, to himself and the environment". Psychotherapy of patients is aimed at changing inadequate reactions to the disease, creating realistic attitudes towards treatment, restoring intrafamilial and broader social ties and, thereby, contributes not only to improving their condition, but also to preventing relapse of the disease, while achieving these psychotherapeutic goals is possible only with taking into account the change in attitudes towards the disease.

Most authors identify at least three factors that influence the formation of attitudes towards the disease: premorbid personality traits, the nature of the disease itself, and socio-psychological schemes.

Consideration of the attitude to the disease from the standpoint of psychology, by its very nature, includes an analysis of all three of the above factors. The attitude towards the disease, like any attitude, is individual, selective, conscious (or capable of awareness), that is, it reflects the individual or personal level. It is subjective and objective in nature, is meaningful and cannot be considered outside the object of relations, in other words, it is determined by the nature of the disease itself. And, finally, like any attitude, attitude to the disease is mediated by the existing in the significant for the patient microsocial environment and in society as a whole, ideas about the disease, about the norms of patient behavior regulated in a certain culture, about the social and sociopsychological consequences of the disease $[1,2]$.

It is important to remember that the attitude towards the disease stands out as an element of the psychological analysis of the integral system of relations and therefore cannot be considered in isolation. In addition, the patient's attitude to his disease is significant and, therefore, influences other relationships of the personality. Thus, for a comprehensive study of the attitude towards the disease, it is necessary to consider it in a broader context, taking into account also the attitude to those areas of the functioning of the individual, which can be influenced both by the fact of the disease itself and the attitude of the individual towards it. The attitude to the disease, like every psychological attitude, is individual and unique. 
However, psychological analysis shows that the attitude of a particular person, while remaining unique, can be described through the belonging of this attitude to certain psychological types of relationships, that is, through identifying similarities with the relationships of other people that are unique in nature. This statement is also true for the description of the attitude to the disease. The typological approach to the description of psychological relations allows the use of methods of scientific generalization for concise transmission of the most essential information about the attitude of a particular patient to his illness. This, in turn, enables the practicing clinical psychologist and the attending physician to use this information to build an optimal psychotherapy algorithm that can significantly influence the patient's condition $[3,4]$.

Currently, the main criteria for the treatment and rehabilitation effectiveness are traditional medical and biological parameters, but they do not reflect the nature of the patient's well-being, the success of their functioning in everyday life and the quality of life in general. In accordance with one of the most important principles of rehabilitation - the personal approach - it is mental adaptation that allows you to maintain a dynamic balance (homeostasis) of the organism with the environment and establish effective relationships with the social environment. From the standpoint of the modern complex neuro-rehabilitation approach, the patient is no longer a passive object of influence, but participates in the treatment and rehabilitation process as its active subject together with the doctor. Features of the patients' personality and their position in relation to a disease become important factors of successful sociopsychological adaptation and rehabilitation.

With the development of oncology, along with traditional methods of treatment, the issues of the psychoemotional state of patients have become especially relevant. "... Surgery is not limited to science and technology. Painfully affecting the human body, penetrating deeply into it, surgery reaches the heights of its capabilities only if it is adorned with the highest manifestations of disinterested care for a sick person and, moreover, not only about his body, but also about the state of his psyche. Not everyone understands this, they teach it too little",-Petrov said back in 1945. It became obvious that malignant tumors or cancer are one of the diseases that carry a powerful stress load and in these conditions, one of the important psychological problems in oncological practice is the problem of the personality's response to the disease [5]. The overall success of treatment depends on how the patient treats his disease. Information about an oncological disease disrupts the normal and safe existence of a person and fills his life with difficult experiences, the core of which is the fear of death. As G.I.Rossolimo wrote: "Any suffering and illness introduces such changes into the spiritual world of a person, putting forward some of its sides, obscuring others, sometimes changing the whole harmony of the personality, as well as the nature of relations to oneself and to everything around, which is the basis to conclude that a doctor in his activity has to reckon not with an ordinary person, but with a suffering person as a special psychological variety" [6].

Rehabilitation of a neuro-oncological patient is understood not only as eliminating the disease itself (the prerogative of treatment) and restoring the physical functions of the body, but also as the most acceptable restoration of a person's ability to live in his usual environment. In the words of NN Petrov: "The patient is always a human personality, with all its complex experiences, and not by no means an impersonal case". Features of the patients' personality and their position in relation to a disease become important factors of successful socio-psychological adaptation and rehabilitation.

Subjective assessment of the patient's condition is the basis of the internal picture of the disease (IPD) - a clinical and psychological phenomenon, which is a special form of psychological adaptation and the leading subsystem of human behavior regulation. The concept of the IPD internal picture of the disease comprises "everything that a patient feels and is worried about, the whole mass of sensations, not only local pains, but overall health, introspection, ideas about the illness, about its reasons - everything that is related to the patient's coming to the doctor, the whole vast inner world of the patient, which consists of very complex combinations of perceptions and sensations, emotions, affects, conflicts, mental experience and traumas" [7]. In these conditions, the correction of the position in relation to one's illness with the use of psychological means inevitably leads to an improvement in the condition, prevention of relapses, relief of the severity of suffering and relief of anxiety. The doctor, taking into account the patient's IKB, always has the opportunity to more fully understand the etiology and pathogenesis of the disease, to adjust the course of treatment, based on the characteristics of the patient's personality $[8,9]$.

Subjective experience of the fact of detection of neurooncological disease and subsequent surgical intervention are among the emergency psychotraumatic stressors, resulting in development of posttraumatic stress of varying severity with most patients [10]. The identification of the type of IPD formed under its influence allows to optimize the strategy of neurorehabilitation measures at the expense of psychotherapeutic correction of maladaptive personal reactions.

When describing the clinical picture of oncology patients, it is customary to distinguish two main psychological variants reflecting the polar types of the internal picture of the disease - hypernozognosic and hyponozognosic [11].

The former is characterized by a high significance of the experience of bodily distress and the conviction that it is impossible to cope with the disease on their own. In the clinical picture anxiety-depressive and hypochondriac experiences come to the fore, the frequency of requests for medical care sharply increases, there is a desire for active use of alternative medicine and unjustified combination of drugs. The latter type stems from devaluation and ignoring somatic symptoms and naive 
conviction in spontaneous "natural" recovery ("it will pass by itself"). Such patients are characterized by untimely treatment for medical care, failure to comply with medical recommendations and prescriptions, refusal of course therapy, gross violations of the treatment regimen. Pronounced manifestations of both hypernozognosia and hyponozognosia have a serious negative impact on the effectiveness of all stages of treatment and diagnostic process [11].

The aim of the research was to study the attitude of patients operated on for pituitary adenoma of their disease and the types of response to the disease, as well as the impact of the type of IPD on the success of the treatment and rehabilitation process.

\section{MATERIALS AND METHODS}

The study involved 52 patients (24 men and 28 women) aged 32-46 years (average age 37,7 $\pm 1,2$ years), operated on for pituitary adenoma in neurosurgical departments of hospitals in Rostov-on-Don for the period from January 1, 1988 to December 31, 2007.

The criteria for inclusion in the study were as follows: a verified diagnosis, a period of at least 36 months after surgery, a completed stage of physiological and functional recovery, absence of intensive neurorehabilitation procedures in specialized Russian and foreign clinics, absence of neurological symptoms due to other topical diseases of the nervous system, absence of severe somatic pathology.

The following methods and techniques were used in the medical and psychological research:

- neurological examination of patients;

- analysis of medical records (medical history, outpatient cards);

- semi-structured interviews aimed at clarifying information about the socio-psychological and labor adaptation, circumstances of life and illness of patients;

- patient assessment using assessment scales (Neuropsychiatric Assessment Scale, Rappaport Impairment Scale, Bartel Index, Hospital Anxiety and Depression Scale, Galvestone Orientation and Amnesia Test, Glasgow Fate Scale);

- analysis of the results of additional research methods conducted during examination (MRI, CT, EEG, ultrasound of extracranial vessels, hormonal parameters, blood biochemical parameters, consultations of related specialists).

For subjective assessment of the success/failure of patients' adaptation during the postoperative period we used: methods of diagnostics of social and psychological adaptation of C. Rogers and R. Diamond (SPA), methods for psychological diagnostics of attitudes to the disease "TOBOL" (Wasserman L. I. et al., 2005), Giessen questionnaire of somatic complaints (in adaptation by Ababkov V. A. et al., 1993). Statistical processing, creation of a database of the obtained data, construction of graphs and diagrams were carried out on a personal computer using the Statistica for Windows version 8.0 application package (StatSoft, USA), Microsoft Office Excel 2007, Biostat (Glantz S., 1999), SPSS 13.0. The procedure for statistical processing of the obtained empirical data included several stages. When comparing groups among themselves according to a quantitative criterion according to the Kolmogorov-Smirnov criterion, the hypothesis about the type of distribution of the trait in groups, correspondence to the normal distribution law, was initially tested. If the variances of the studied trait in the groups were equal (the test was carried out using analysis of variance), parametric tests were used to compare the groups for this trait - Student's t-test when comparing two groups, Fisher's test in multiple comparisons with an assessment of the confidence probability of these criteria. If the assessment of the initial data by the type of distribution did not allow the use of parametric research methods, since the distribution of data did not obey the normal law, the determination of the reliability of differences between the compared groups was carried out using the Mann-Whitney test, Pearson's chi-square using the standard formula, taking into account the Yates correction for paired comparisons. All differences were considered significant at $\mathrm{p}<0.05$. The level of cognitive and mnestic functions with all patients did not reveal significant differences $(p>0,05)$, which allowed to include a group of the specified number $(n=52)$ in this study.

\section{RESEARCH RESULTS}

During neurological examination a certain neurological deficit was diagnosed (table I). When comparing the neurological status of patients in the early postoperative period and at the time of the current examination, a significant regression of neurological symptoms during the study period was revealed $(\mathrm{p}<0,05)$.

As can be seen from the table, in the overwhelming majority of patients after surgery, visual and endocrine disorders were preserved, in some of them they appeared immediately after surgery and radiation therapy [12]. However, despite the fact that most of the identified disorders had no significant severity and did not interfere with their social and labor (professional) adaptation, all patients had II (38 people/73,1\%) and III (14 people $/ 26,9 \%$ ) disability classes. The majority of patients (41 people/78,8\%) referred themselves to the group of "disabled" and "in need of assistance".

Attention was drawn to the fact that in the study group of patients at the time of examination objective clinical manifestations were accompanied by the presence of significantly pronounced subjective symptoms (table II). Almost all patients, as a rule, complained of headaches, dizziness, noise in the head and ears, pain and discomfort in the heart, sleeping disorders, general weakness, rarely double vision, pain in the limbs, muscles, joints, a significant number of patients demonstrated frequent psychovegetative paroxysms. In this regard, in the vast majority of cases, patients (50 people/96,2\%), dismissed after surgical treatment, were forced to abandon active work or change their professional affiliation in accordance with the available opportunities without being deeply disabled. 
Table I . Objective neurological symptoms observed in patients operated for pituitary adenoma at the time of initial examination

\begin{tabular}{|c|c|c|c|c|c|c|}
\hline \multirow[b]{2}{*}{ Symptoms } & \multicolumn{2}{|c|}{ Male } & \multicolumn{2}{|c|}{ Female } & \multicolumn{2}{|c|}{ Total } \\
\hline & & $\%$ & & $\%$ & & $\%$ \\
\hline Unilateral amaurosis & & 0 & & 7,1 & 2 & 3,9 \\
\hline Amblyopia & 6 & $25,0 *$ & 3 & 10,7 & 9 & 17,3 \\
\hline $\begin{array}{l}\text { Combination of amaurosis and } \\
\text { amblyopia }\end{array}$ & 2 & 8,3 & 6 & $21,4 *$ & 8 & 15,4 \\
\hline Bitemporal hemianopsia & 0 & 0 & 1 & 3,6 & 1 & 1,9 \\
\hline Endocrinal disorders & 6 & $25,0 *$ & 2 & 7,1 & 8 & 15,4 \\
\hline III n deficit, liquorrhea & 1 & 4,2 & 0 & 0 & 1 & 1,9 \\
\hline Optic nerve atrophy, trophic disorders & 1 & 4,2 & 4 & $14,3 *$ & 5 & 9,6 \\
\hline $\begin{array}{l}\text { Combination of visual and endocrinal } \\
\text { disorders }\end{array}$ & 8 & $33,3 *$ & 10 & $35,7^{*}$ & 18 & 34,6 \\
\hline Total & 24 & 100,0 & 28 & 100,0 & 52 & 100,0 \\
\hline
\end{tabular}

Note: $\mathrm{p}<0.05$ compared to the total number of patients

Table II. Complaints made by patients operated on for pituitary adenoma at the time of initial examination

\begin{tabular}{|c|c|c|c|c|}
\hline Symptoms/complaints & $\begin{array}{l}\text { Number of } \\
\text { complaints } \\
\text { (male) }\end{array}$ & $\begin{array}{l}\text { Number of } \\
\text { complaints } \\
\text { (female) }\end{array}$ & $\begin{array}{l}\text { Total complaints } \\
\text { made by patients }\end{array}$ & $\begin{array}{c}\% \text { of } \\
\text { complaints } \\
\text { from the } \\
\text { number of } \\
\text { examined } \\
\text { patients }\end{array}$ \\
\hline Headache & & $22 *$ & 40 & 76,9 \\
\hline Dizziness & 2 & $16^{*}$ & 18 & 34,6 \\
\hline Pain in the heart & 8 & 20 & 28 & 53,8 \\
\hline Noise in the head/ears & $6^{*}$ & 1 & 7 & 13,5 \\
\hline Pain in the limbs & 4 & $18^{*}$ & 22 & 42,3 \\
\hline Sleeping disorder & 16 & $20 *$ & 36 & 69,2 \\
\hline Attacks of suffocation (isolated) & 4 & $6^{*}$ & 10 & 19,2 \\
\hline Heart attacks (isolated) & $6^{*}$ & 4 & 10 & 19,2 \\
\hline $\begin{array}{l}\text { Developed psychovegetative } \\
\text { seizures (typical and atypical panic } \\
\text { attacks) }\end{array}$ & 6 & $18^{*}$ & 24 & 46,2 \\
\hline Total subjects & 24 & 28 & 52 & - \\
\hline
\end{tabular}

Note: $\mathrm{p}<0.05$ compared to the total number of patients

To verify the system of personal attitudes associated with the disease, the study used the technique of "TOBOL" [13], based on the classification of attitudes by A.Y. Lichko and N. Y. Ivanov. This test procedure allows to diagnose the leading type of patient's response to the disease and treatment:

- sensitive, characterized by excessive vulnerability, vulnerability, preoccupation with possible unfavorable impressions that information about the disease can produce on others, fear of becoming a burden for loved ones due to illness and an undesirable attitude on their part in this regard, mood swings, mainly associated with with interpersonal contacts;
- anxious, (anxious-depressive and obsessivephobic) with a predominance of continuous anxiety and suspicion about the unfavorable course of the disease, possible complications, ineffectiveness and even the danger of treatment with the search for new methods of treatment, additional information about the disease and methods of therapy with frequent changes in the attending physician;

- hypochondriacal, based on excessive concentration on subjective painful and other unpleasant sensations, with exaggeration of real and looking for non-existent diseases and suffering, the desire to constantly tell doctors, medical staff and others about them with a 
combination of desire to be treated and lack of faith in success;

- melancholic (vitally melancholy) due to an overcompulsive illness, a pessimistic view of everything around with active depressive statements up to suicidal thoughts, disbelief in recovery, possible improvement and effectiveness of treatment, even with favorable objective data and satisfactory health;

- apathic, characterized by complete indifference to one's fate, a loss of interest in life, in everything that previously worried, the outcome of the disease and the results of treatment with passive submission to procedures and therapy with persistent prompting from the outside;

- neurasthenic with behavior of the type of "irritable weakness", with outbursts of irritation, inability and unwillingness to endure painful sensations, outbreaks of irritation, followed by a critical attitude to their actions, thoughtless words and requests for forgiveness;

- egocentric (hysterical), which is based on the "acceptance of the disease" and the search for benefits, in this regard, with the exposure of relatives and others to their sufferings and experiences in order to evoke sympathy and completely seize their attention, the requirement of exclusive concern for oneself to the detriment of other matters against the background of complete inattention to other people, emotional instability and unpredictability;

- paranoid, due to the belief that the disease is the result of external causes, someone's malicious intent with extreme suspicion and vigilance about talking about oneself, drugs and procedures, the desire to attribute possible complications or side effects of therapy to the negligence or malicious intent of doctors and staff, accusations and requirements for punishment in connection with this;

- anosognosic (euphoric), which is based on the active rejection of the thought about the disease and its possible consequences, up to the denial of the obvious with the refusal of medical examination and treatment in violation of the regimen and medical recommendations against the background of an unreasonably elevated mood, a scornful frivolous attitude to the disease and therapy;

- dysphoric (aggressive) in the structure of which an angry gloomy, embittered mood dominates, a constant gloomy and displeased look, outbursts of anger with a tendency to blame others for their illness, envy, aggressiveness and hatred of healthy people, including relatives and friends;

- ergopathic (stenic) with "withdrawal from the disease to work" with an overly responsible, sometimes obsessive attitude towards it, the desire at all costs to maintain a professional status and the ability to continue active work in the same quality, combined with a selective choice of methods of examination and treatment; and, finally, harmonious (realistic, balanced), characterized by a real assessment of one's condition without a tendency to exaggerate or underestimate its severity against the background of a desire to actively contribute to the success of treatment in everything and to alleviate the burden of caring for loved ones.

The "TOBOL" methodology, built in the form of a questionnaire, diagnoses the type of attitude towards the disease based on information about how the patient relates to a number of life problems and situations, potentially the most significant for him and directly related to his disease. These relations of the patient were studied as 12 subsystems in the general system of personality relations. These subsystems determine the structuring of the content of the methodology according to 12 topics: attitude to the disease, to its treatment, doctors and medical staff, relatives and friends, work (study), loneliness, the future, as well as self-esteem of well-being, mood, sleep and appetite. The questionnaire includes, respectively, 12 tables-sets of statements ("feeling", "mood" and others). Each, in turn, contains from 10 to 16 statements proposed to the subject, drawn up on the basis of the clinical experience of a group of experts. When working with the technique, the patient is asked to select the two most appropriate statements for each topic. The tables also contain one additional sentence: "None of the statements suit me." This form of the questionnaire better reveals such a system of relations than working with a questionnaire, which uses the answers "yes" or "no", as it allows to identify the most significant characteristics of attitudes towards the disease. Rules for the subject's work with the questionnaire: the subject is asked to select two statements that are most suitable for him in each of the table-set and circle the numbers of the choices made in the registration sheet. If the patient cannot choose two statements on any topic, he must mark the last statement in the corresponding tableset. The time for filling out the registration sheet is not limited. The study can be carried out simultaneously with a small group of subjects, provided that they do not consult with each other. In addition, data about the patient is entered in the registration sheet in accordance with the practical and research tasks facing the doctor and clinical psychologist, such as: a detailed clinical diagnosis and leading syndrome, duration of the disease, disability, prognosis of the disease, changes in social and family status due to with the disease. The subject's protocol can be characterized by the sums of diagnostic coefficients, each of which corresponds to a certain type of attitude towards the disease and can be considered as a separate scale. In general, the profile of 12 psychodiagnostic scales characterizes the totality of individual aspects of the patient's multidimensional attitude to his disease and is subject to interpretation by analogy with other psychological techniques. These 12 types of attitudes are combined into 3 blocks according to two criteria: "adaptability-maladaptivity", which reflects the influence of attitude to the disease on the adaptation of the patient's personality, "interpsychic-intrapsychic orientation" of maladaptation.

The first type includes harmonious, ergopathic and anosognosic types of attitude to the disease, in which mental and social adaptation is not significantly disturbed. The second type includes types of attitude to the disease, causing mental maladaptation and having mainly intrapsychic orientation of reactions to the disease (anxious, hypochondriac, neurasthenic, melancholic and apathetic types of attitude). The third type combines types of attitude to the disease, which also lead to 
maladaptation, but are characterized by interpsychic orientation of the response, that is, manifested primarily in interpersonal interaction (sensitive, egocentric, dysphoric and paranoid types of attitude).

The application of the "TOBOL" technique allowed to obtain a detailed description of the system of personal attitudes of patients operated on for pituitary adenoma and to determine the holistic type of response to the disease. Total results of the examined patients are presented in table III.

Table III. Details of the internal picture of the disease of patients operated on for pituitary adenoma.

\begin{tabular}{|l|c|c|c|c|c|c|}
\hline \multirow{2}{*}{ Type of attitude to the disease } & \multicolumn{2}{|c|}{ Male } & \multicolumn{2}{c|}{ Female } & \multicolumn{2}{c|}{ Total } \\
\cline { 2 - 7 } & & & & & & \\
& & & & & & \\
\hline Harmonious & & 0 & & 0 & & 0 \\
\hline Ergopathic & 3 & $12,5^{*}$ & 1 & 3,6 & 4 & 7,7 \\
\hline Anosognosic & & 0 & & 0 & & 0 \\
\hline Anxious & 2 & 8,3 & 5 & $17,9^{*}$ & 7 & 13,5 \\
\hline Hypochiondrial & 5 & $20,8^{*}$ & 4 & 14,3 & 9 & 17,3 \\
\hline Neurasthenic & 3 & $12,5^{*}$ & 3 & 10,7 & 6 & 11,5 \\
\hline Melancholic & 1 & 4,2 & 1 & 3,6 & 2 & 3,9 \\
\hline Apathetic & 1 & 4,2 & 4 & $14,3^{*}$ & 5 & 9,6 \\
\hline Sensitive & 3 & $12,5^{*}$ & 2 & 7,1 & 5 & 9,6 \\
\hline Egocentric & 1 & 4,2 & 3 & $10,7^{*}$ & 4 & 7,7 \\
\hline Paranoid & 1 & 4,2 & 3 & $10,7^{*}$ & 4 & 7,7 \\
\hline Dysphoric & 4 & $16,6^{*}$ & 2 & 7,1 & 6 & 11,5 \\
\hline Total & 24 & 100,0 & 28 & 100,0 & 52 & 100,0 \\
\hline
\end{tabular}

The data given in the table III indicate that the "typical" patient operated on for pituitary adenoma is characterized by a complete absence of harmonic (adequate) and anosognosic types of the IPD. Attention is drawn to the minimum severity ( 4 people/7,7\%) of the ergopathic variant of the attitude to the disease, focused on overcoming the disease and the expressed desire to maintain the same level of social activity.

A distinct dominance of emotionally saturated hypernosognosic variants of response to the disease with a predominance of obsessive fears, irritable weakness, pessimistic assessment of the prospects, having mainly intrapsychic orientation (i.e. causing suffering to patients themselves), was revealed in 29 people (55,8\% of cases). Among these response options the highest proportion belongs to the hypochondriac type of the IPD (9 people/17,3\%), which is demonstrated in table II. In particular, this type of response is characterized by excessive concentration of patients on their subjective painful sensations. Such patients complain to others about the state of their health, often aggraving their own suffering in search of a caring and sympathetic attitude to themselves. Due to the fear of "missing something very important" and significant for the correct understanding of their condition, such patients describe their state of health with excessive detail. Remarks of the immediate environment concerning exaggeration of severity of the given disease, compensation neurosis (a wish to gain a secondary benefit from illness) and mistrust to complaints, as a rule, cause an extremely negative, aggressive reaction in them. A distinctive feature of this type of the IPD is an extremely ambivalent combination of the desire to be treated and disbelief in the success of treatment, the requirements of a thorough examination and a momentary fear of harmful and painful procedures.
Continuous anxiety and suspiciousness of patients, confidence in the unfavorable course of the disease, possible complications are characteristic of the diagnosed anxious type of attitude to the disease. This type of response to disease and treatment was detected in 7 people $(13,5 \%$ of the total sample). Typical manifestations of this anxious type included an increased interest in the medical literature in connection to search for additional information, objective data about the disease and new methods of treatment. At the same time such patients are often sharply critical of the medical staff, seek to double-check the information received from doctors about their disease, as they are extremely worried that their usual lifestyle and activities will change in connection with the disease.

Moreover, the prevalence of the neurasthenic type of response was revealed in the examined group of patients. Such patients numbering 6 people $(11,5 \%$ of cases) in their statements noted subjective intolerance of pain, impatience and intemperance with respect to their condition. The behavior of representatives of this type of IPD is realized as "irritable weakness" with outbreaks of verbal aggression, anger in response to unpleasant sensations and/or pain, to the need for examination and recommended procedures. Often such reactions of the displaced aggression type "pour out" on the immediate environment. Subsequently, due to feelings of guilt and the onset of remorse, patients often begin to implement strategies of apologetic behavior.

The last most pronounced type of the IPD for the examined group of patients operated on for pituitary adenoma was the dysphoric type (6 people / 11,5\%). This variant of personal response, like the previous type of anxiety, leads to maladaptation, but is characterized primarily by interpsychic orientation. These are patients 
with a heavy gloomy appearance, with a constant gloomy and embittered mood, envious and hostile to healthy people. Qualitative characteristics of the representative of this type are as follows: outbursts of anger towards others, the tendency to blame others (especially the loved ones) in their illness and its consequences. Such patients are painfully demanding, suspicious and distrustful of both procedures and treatment, waiting for the next "trick" of the medical staff and life itself. Very often such patients turn into domestic tyrants in the family environment.

\section{CONCLUSION}

The study of patients operated on for pituitary adenoma allows us to state a high level of nosogenic stress reactions, largely explained by the ongoing psychotraumatic situation of the neurooncological disease. The revealed variants of the internal picture of the disease of the "typical" representative of this cohort of patients indicate a significant predominance of maladaptive attitude to the disease and treatment (48 people $/ 92.3 \%$ as opposed to the adaptive, namely ergopathic type - 4 people/7,7\%) and a mainly intrapsychic orientation $(p<0,05)$. Diagnosed dominance of maladaptive types of the IPD significantly reduces the effectiveness and prevents the success of postoperative neurorehabilitation of patients.

Thus, the results indicate the need for mandatory inclusion of a complex of psychological and psychotherapeutic measures for the correction of maladaptive personal reactions and qualitative restructuring of the system of personal relationships in order to maximize the recovery of the physical, psychological and social status of patients with pituitary adenoma.

\section{REFERENCES}

[1] T.A. Karavaeva, V.A. Mikhailov, A.V. Vasilyeva, S.V. Poltorak, A.Yu. Polyakov, M.V. Razina, and N.Yu. Safonova, "Development of a comprehensive personalized program for the correction of dyssomnic disorders in the structure of neurotic disorders," Neurological Bulletin. Journal them. V.M. Bekhtereva, vol. XLIX, issue 2, pp. 31-36, 2017.

[2] C.M. Morin, J.R. Davidson, and S. BeaulieuBonneau, "Cognitive Behavior Therapies for Insomnia I: Approaches and Efficacy," in Principles and Practice of Sleep Medicine, 6th ed., M.H. Kryger, T. Roth, W.C. Dement, Eds. Philadelphia, US: Elsevier, 2016, pp. 804-813.

[3] E.A.F. Holmes, and D.A. Hughes, "Predicting Adherence to $\mathrm{M}$ edications Using Health Psychology Theories: A Systematic Review of 20 Years of Empirical Research," Value In Health, vol. 17, pp. 863-867, 2017.

[4] T.Yu. Semiglazova, G.A. Tkachenko, and V.A. Chulkova, "Psychological aspects of the treatment of cancer patients," Malignant tumors,vol. 4, special issue 1, pp. 54-58, 2016.

[5] N.E. Vodopyanova, Psychodiagnostics of stress. Saint Petersburg, Russia: Peter, 2009, p. 336.
[6] A.P. Karitskiy, V.A. Chulkova, E.V. Pesterova, and T.Yu. Semiglazova, "Rehabilitation of a cancer patient as the basis for improving the quality of his life," Problems of Oncology, vol. 2, pp. 180-184, 2015.

[7] R.A. Luria, Internal picture of the disease and iatrogenic diseases, 4th ed., Moscow, Russia: Medicine, 1977, p. 112.

[8] T.A. Karavaeva, A.V. Vasilyeva, T.Yu. Semiglazova, A.P. Karitsky, E.P. Lukoshkina, and A.M. Belyaev, "Algorithm for the diagnosis of neurotic level anxiety disorders in cancer patients," Questions of oncology, vol. 2, pp. 355-360, 2016.

[9] E.B. Mizinova, T.A. Karavaeva, S.V. Poltorak, and R.M. Belan, "Irrational cognitive attitudes of patients with anxiety-phobic neurotic disorders as targets of psychotherapy," Review of psychiatry and medical psychology, vol. 1, pp. 53-58, 2017.

[10]M.S. Kurchakova, and N.V. Tarabrina, "Psychological protection and oncology psychophysiological approach," in Proc. I International Congress "Psychosomatic Medicine 2006", Saint Petersburg, 2006, p. 121.

[11]A.V. Andryushchenko, "Basic principles of rehabilitation of cancer patients," in Proc. VI AllRussian Congress of Oncologists, Moscow, 2005, pp. 39-40.

[12] J.A. Jane, and E.R. Laws, "Surgical Management of Pituitary Adenomas," Singapore Med J., vol 43(6), pp. 318-323, 2002.

[13]L.I. Wasserman, B.V. Iovlev, and E.B. Karpov, Psychological diagnostics of attitudes towards illness. Saint Petersburg, Russia: Publishing house of the NIPNI named after V.M. Bekhterev, 2005, p. 32.

\section{Creative Commons Attribution License 4.0 (Attribution 4.0 International, CC BY 4.0)}

This article is published under the terms of the Creative Commons Attribution License 4.0 https://creativecommons.org/licenses/by/4.0/deed.en US 